\title{
A 6-bit 2GS/s CMOS Time-Interleaved ADC for Analysis of Mixed-Signal Calibration Techniques
}

\author{
Benjamín T. Reyes ${ }^{\ddagger}$, Lucas Tealdi ${ }^{\dagger}$, German Paulina ${ }^{\dagger}$, \\ Emanuel Labat ${ }^{\dagger}$, Raúl Sanchez ${ }^{\dagger}$, Pablo S. Mandolesi*, and Mario R. Hueda ${ }^{\ddagger}$ \\ $\ddagger$ Laboratorio de Comunicaciones Digitales - Universidad Nacional de Córdoba - CONICET \\ Av. Vélez Sarsfield 1611 - Córdoba (X5016GCA) - Argentina \\ $\dagger$ Fundación Fulgor - Romagosa 518 - Córdoba (5000) - Argentina \\ * GISEE - LMNE - Universidad Nacional del Sur - Av. Alem 1253 - Bahía Blanca - Argentina \\ Emails: \{breyes, mhueda\}@efn.uncor.edu
}

\begin{abstract}
A 6-bit 2-GS/s time interleaved (TI) successive approximation register (SAR) analog-to-digital converter (ADC) is designed and fabricated in a $0.13 \mu \mathrm{m}$ CMOS process. The architecture uses 8 time-interleaved track-and-hold amplifiers (THA), and 16 SAR ADC's. The chip includes $(i)$ a programmable delay cell array to adjust the interleaved sampling phase, and (ii) a 12 Gbps low voltage differential signaling (LVDS) interface. These blocks make the fabricated ADC an excellent platform to evaluate mixed-signal calibration techniques, which are of great interest for application in high-speed optical systems. Measurements of the fabricated ADC show $33.9 \mathrm{~dB}$ of peak signal-to-noise-and-distortion ratio (SNDR) and $192 \mathrm{~mW}$ of power consumption at $1.2 \mathrm{~V}$.
\end{abstract}

\section{INTRODUCTION}

Time-interleaved analog-to-digital converters (TI-ADC) are widely used to provide high-sampling rate for digital receivers architectures (e.g., wireline and optical links) [1]. A basic TIADC architecture includes $M$ single converters (or channels) operating in a parallel fashion at frequency $1 / T$ but with different sampling phases in order to achieve an overall sampling rate of $M / T$. It is well known that TI-ADC's are sensitive to mismatches of the offset, gain, and sampling phase among the channels. These impairments are collectively known as fixedpattern noise (FPN).

Numerous calibration techniques for FPN compensation have been proposed [2]-[6]. Recently, mixed-signal calibration techniques have received special attention for applications in ultra-high speed optical communication systems [4], [5], [7]. For example, the minimization of the mean squared error (MSE) or the bit-error rate (BER) by using a gradient algorithm has been proposed in [8]. Typically, long computer simulation run time is required to evaluate the performance of mixed-signal calibration algorithms such as the one described in [8]. We highlight that this problem is exacerbated when the communication system under analysis includes complex algorithms such as powerful forward error correction (FEC) codes. In this context, emulation based on field-programmable gate array (FPGA) platforms are usually adopted to save time. From the above, it can be inferred that the design and fabrication of an integrated high-speed TI-ADC that includes $(i)$ capabilities to adjust the sampling phase among the channels

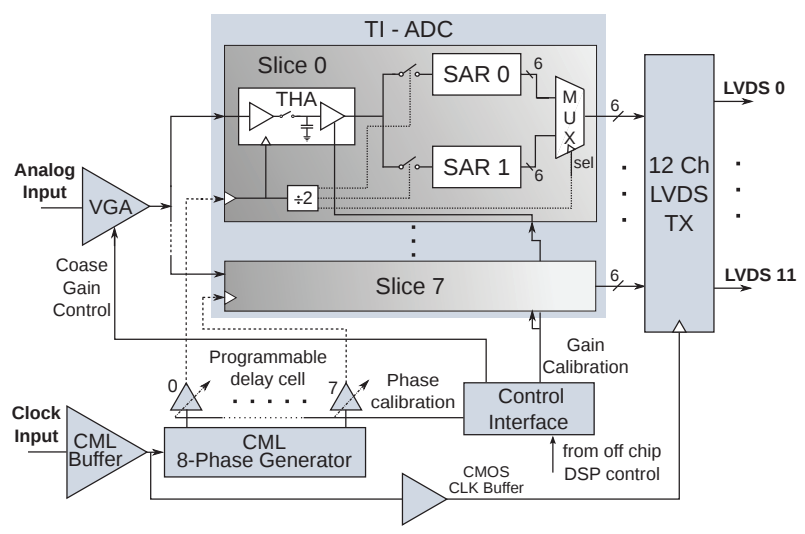

Fig. 1. Time-Interleaved ADC chip architecture.

and (ii) a multigigabit interface to connect with powerful FPGA platforms is of great interest to design mixed-signal calibration algorithms for next-generation optical networks.

This paper presents a 6-bit 2-GS/s time-interleaved successive approximation register (SAR) ADC. The architecture uses 8 time-interleaved track-and-hold amplifiers (THA), and 16 SAR ADC's. The chip includes (i) a programmable delay cell array to adjust the interleaved sampling phase, and (ii) a 12 Gbps low voltage differential signaling (LVDS) interface. The designed ADC can be used to save time during the design of mixed-signal calibration techniques [8]. The ADC fabricated in a $0.13 \mu \mathrm{m}$ CMOS process achieves $33.9 \mathrm{~dB}$ of signal-to-noise-and-distortion ratio (SNDR) and $192 \mathrm{~mW}$ of power consumption at $1.2 \mathrm{~V}$.

The rest of this paper is organized as follows. Section II describes the chip architecture. Section III presents the design of the asynchronous SAR ADC and details of the comparator offset calibration circuit. Section IV shows results from measurements of the fabricated ADC. Finally, concluding remarks are drawn in Section V.

\section{Architecture of ThE TI-ADC}

The chip architecture is shown in Fig. 1. Next we present the design requirements and details of the architecture of the fabricated TI-ADC. 


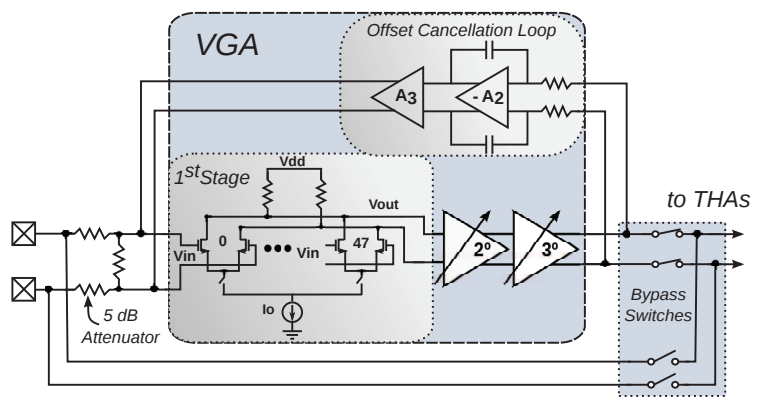

Fig. 2. Variable gain amplifier (VGA) composed by a 3-stage differential amplifier with digital gain control.

\section{A. Requirements}

This $\mathrm{ADC}$ is required to be the core of a test/verification platform for time skew mismatch calibration algorithms in time interleaved ADCs. Towards this end, the design includes eight interleaved THA and sixteen single SAR converters to achieve an overall high sampling rate such as $2 \mathrm{GHz}$. The main requirement from the point of view of the algorithm verification concept is a wide flexibility of clock phase control in each THA. The sampling frequency of the TI-ADC can be set from $200 \mathrm{MHz}$ to $2 \mathrm{GHz}$. Furthermore, the chip has to be able to vary around a $\pm 25 \%$ of the sampling clock phase in each THA for all frequency range. This means that each delay cell has to have a wide programmable delay range to meet the specification. In addition, the ADC test chip has to transmit all the samples without decimation, thus the complete information can be used on an off-chip processor.

\section{B. Input Variable Gain Amplifier}

The analog input signal of the chip is buffered with a digitally controlled variable-gain amplifier (VGA). This block is required to allow an automatic gain control (AGC) of the input signal for optimum dynamic range. The VGA has three active stages (Fig. 2) and each stage consists of 48 differential pairs connected in parallel with an offset cancellation loop [9]. The gain of the stages is $A_{v} \propto g_{m}$, where $g_{m}$ is the MOS transconductance. Then, turning on/off each differential pair of each stage, the number of active MOS is changed then $g_{m}$ (and $A_{v}$ ) can be controlled. The total current and load resistor of each stage of the VGA is kept constant, thus the output common-mode voltage does not change. Moreover, the analog input is first connected to a passive attenuator before connecting to VGA in order to reduce distortion. If the gain control is not required, the VGA can be bypassed with MOS switches therefore the VGA distortion can be avoided.

\section{Time-Interleaved ADC}

The core of the chip is a hierarchical interleaved ADC (see Fig. 1). It includes 8 parallel channels (slices) where each channel consists of one THA and two sub-interleaved SAR ADC. Each THA is managed by a $50 \%$ duty cycle clock at frequency $F_{\text {slice }}=F_{s} / 8=2 \mathrm{GHz} / 8=250 \mathrm{MHz}$. After tracking, the THA turns to the hold mode and the signal is re-sampled alternatively by one of the two SAR ADC for

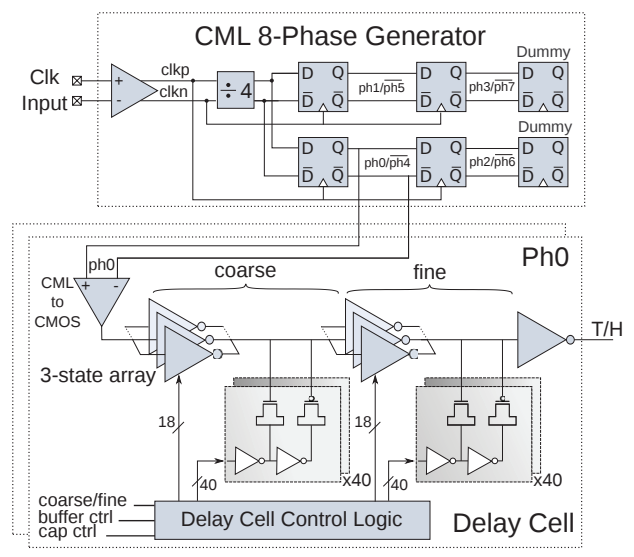

Fig. 3. 8-Phase generator and delay-cell blocks.

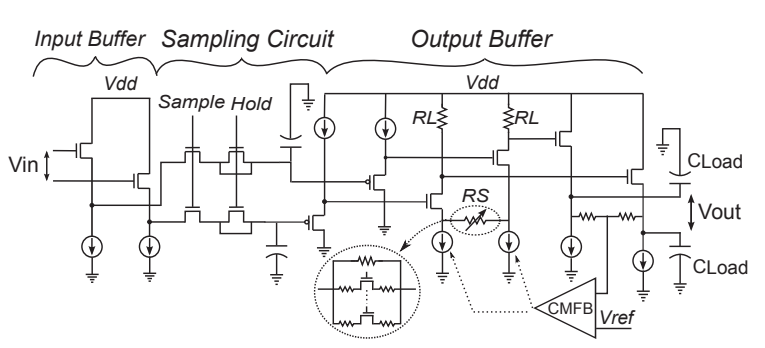

Fig. 4. THA with gain calibration stage.

quantization. The THA and SARs are synchronized by a clock divider that generates two clock signals from THA clock with $25 \%$ duty cycle at $F_{S A R}=F_{\text {slice }} / 2=125 \mathrm{MHz}$. After the quantization, the two SAR outputs are multiplexed to provide a single 6 bits output at $F_{\text {slice }}$ rate.

The eight THA clock phases are provided by a multiplephase clock generator as depicted in Fig. 3. It is based on a double shift-register (SR) therefore an $1 \mathrm{GHz}$ clock input is needed from an off-chip generator in order to achieve $2 \mathrm{GHz}$ sampling rate. The shift registers are implemented in currentmode-logic (CML) to minimize the clock jitter coupled from power supply. Moreover, a CML clock divider is needed to provide the clock pattern at the SR input. In addition, the CML divider is optimized to operate on a wide range of input clock frequencies (100 $\mathrm{MHz}$ to $1 \mathrm{GHz}$ ) with maximum sensitivity [10].

Finally, the digital slice outputs ( 8 channels $\times 6$ bits) are sent to the transmitter (TX). In the TX, the 48 digital slice outputs are multiplexed by 4 to arrange a 12 parallel LVDS channels interface. Note that, unlike most of reported Gigasampled ADC test chips [4]-[6], in our design the full data rate (12 Giga-bits per second (Gbps)) without decimation is sent off-chip.

\section{Track and Hold Amplifier}

The THA topology is presented in Fig. 4. It is composed of an input buffer stage, a sampling circuit, and an output buffer. The input buffer is a pseudo-differential source follower that drives an NMOS sampling circuit. The input-buffer (plus sampling circuit) bandwidth (BW) is near $4 \mathrm{GHz}$. This overbandwidth at the THA input buffer avoids BW mismatch at 


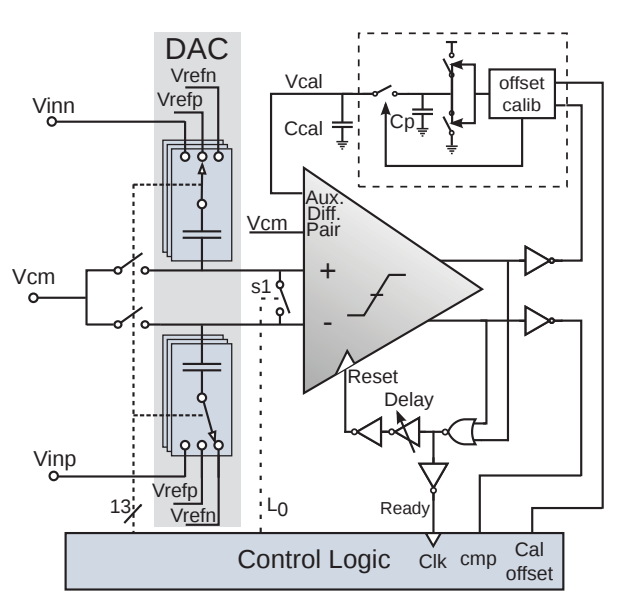

Fig. 5. Asynchronous SAR ADC topology.

Nyquist input frequencies. Moreover, from simulations we verified that the linearity at the sampling circuit is above $60 \mathrm{~dB}$. The output buffer is a 3 stage amplifier where a pseudo-differential common-source degeneration amplifier is used to calibrate the gain mismatch between THAs. The gain is controlled by $R_{L}$ and $R_{S}$ as $A_{v}=R_{L} /\left(1 / g_{m}+R_{S}\right)$. The value of $R_{S}$ is digitally programmed connecting in parallel a fixed resistor with one of the 16 branches of a resistor array.

On the other hand, a last stage source follower buffer is used to drive one of the two SAR digital-to-analog converters (DAC) that alternatively loads the THA. The source follower output stage allows a low SAR DAC settling time $(\tau<150 \mathrm{ps}$ worst case corner) considering a $C_{L}$ equivalent DAC capacitor array of $600 \mathrm{fF}$. Besides, the total harmonic distortion (THD) at the SAR inputs is $-48 \mathrm{~dB}$.

\section{E. Programmable Delay Cell}

The design of digitally programmable delay cells for TIADC phase calibration applications has been widely studied [4]-[6]. Figure 3 shows the delay cells circuit used between the phase generator and the THAs. These cells are able to set a time delay $\left(T_{d}\right)$ to control the relative sampling time between the clock phases. This design considers a fine delay circuit and a coarse delay circuit. The fine delay circuit is used to adjust very small sampling time mismatch. The fine delay range is $T_{d_{\max }}= \pm 0.03 T_{s}$ with 40 steps of $T_{d_{s t e p}}=0.0015 T_{s}$, where $T_{s}=1 / F_{s}$ is the overall sampling period.

As explained in Section II-A, a wide phase control is needed to emulate any relatively large sampling mismatch scenario (e.g., high-speed TI-ADC in optical receivers [8]). Therefore, a coarse delay circuit is implemented with a programmable range of $T_{d_{\max }}= \pm 0.3 T_{s}$ and $T_{d_{\text {step }}}=0.015 T_{s}$. The fine and coarse delay circuit are based on a shunt-capacitor technique where each buffer is loaded by a 40 equal sized MOS capacitor array (MOScap) that is thermometrically switched [6].

Moreover, the ADC can operate from $200 \mathrm{MS} / \mathrm{s}$ to $2 \mathrm{GS} / \mathrm{s}$ with $T_{s}$ varying from $5 \mathrm{~ns}$ to $500 \mathrm{ps}$, respectively. Hence, the delay cell has to be able to vary $T_{d_{\text {step }}}$. The solution for the programmability of $T_{d_{\text {step }}}$ was the addition of a current control which comprises of 18 equal-sized 3-state buffers that allow for the variation of the charge/discharge current of

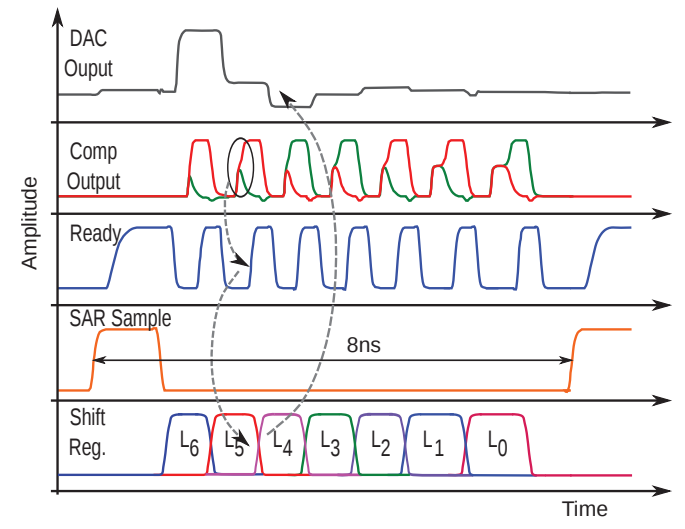

Fig. 6. Asynchronous SAR ADC timing diagram.

the MOScap array. With the combination of both time delay techniques, the coarse $T_{d_{\text {step }}}$ can be set from 7.5 ps to $75 \mathrm{ps}$ and the fine $T_{d_{\text {step }}}$ from 0.75 ps to $7.5 \mathrm{ps}$.

\section{ASYNCHRONOUS SAR ADC}

The asynchronous SAR ADC topology is shown in Fig. 5. It is based on a binary-weighted redistribution-charge DAC, a latched comparator, and a self-clocked asynchronous control logic. The capacitor array is composed of metal-fringe capacitor unit-cells of $6.5 \mathrm{fF}$. The latched comparator used is a PMOS StrongArm [11] with a resistive load differential pre-amplifier. Moreover, the pre-amplifier has an auxiliary differential pair for offset calibration [12].

Figure 6 shows the asynchronous SAR timing diagram. The sequence starts with the SAR sample signal, provided by the internal slice divider and the SAR internal logic (see Fig. 1). Then, the shift-register (SR) is clocked and the DAC is set for MSB comparison. When the comparison ends, a detector (NOR gate at the comparator outputs) toggles the ready signal that is buffered to clock the SR. After that, the control logic sets the DAC and the comparator is reset to allow the next comparison cycle. Moreover, from the critical timing path of control logic, a carefully custom logic implementation is used to achieve high-speed and low power consumption in all the flip-flops and combinational gates.

\section{A. Calibration of the Comparator Offset}

The offset of the comparator defines the SAR offset (without considering the THA offset), and it can take large values (several LSBs) for small area comparators. Therefore, a comparator offset calibration is required to avoid intra-slice offset mismatch (i.e., between the two sub-interleaved SAR) ${ }^{1}$.

As shown in the timing diagram of Fig. 6, after the 6 bits comparison cycles, the signal $L_{0}$ closes the switch $S_{1}$ and the comparator inputs are shorted. Since no input voltage is applied, the next comparison will be decided as a result of the offset voltage at the latch regeneration nodes, including pre-amp and latch offset effects. As proposed in [12], the comparison result is stored as a fixed charge in a small (parasitic) capacitor $C_{p}$ and then it is averaged with the past

\footnotetext{
${ }^{1}$ Note that each THA buffer introduces an inter-slice offset, but this mismatch is compensated with a digital off-chip processing.
} 


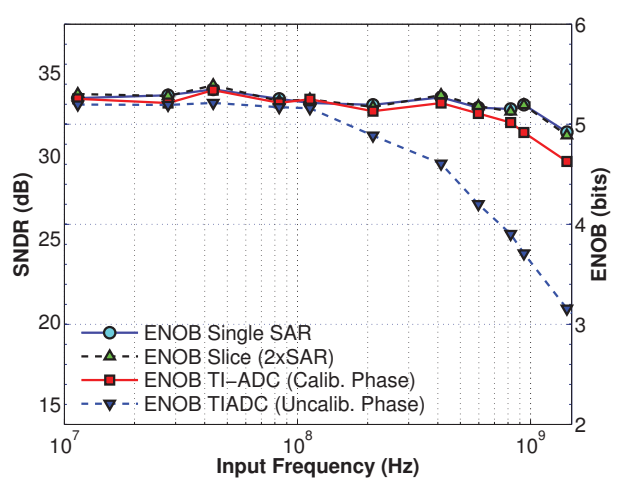

Fig. 7. SNDR and ENOB vs. input frequency.

decisions in an integration capacitor $C_{c a l}$. Finally, the offset calibration loop is closed by the auxiliary differential pair on the pre-amplifier to compensate the equivalent input offset. Note that if there is a comparator metastable state (very long regeneration time) in the approximation cycle, this will not affect the quantization process. If it takes a long time, the offset calibration cycle will be skipped but it will not degrade the offset calibration. A similar scheme has been used recently in [13].

\section{EXPERIMENTAL RESULTS}

The TI-ADC was fabricated in a $0.13 \mu \mathrm{m}$ standard CMOS process and occupies a $1.8 \mathrm{~mm} \times 1.8 \mathrm{~mm}$ area (without including Tx and VGA). In the following, some experimental results of the fabricated TI-ADC with a sampling rate of $F_{s}=2 \mathrm{GS} / \mathrm{s}$ are presented ${ }^{2}$.

Figure 7 shows the SNDR and the effective-number-of-bits (ENOB) as a function of the analog input frequency, $F_{i n}$. The performance of a single SAR converter achieves a peak SNDR of $34.2 \mathrm{~dB}$ (5.39 ENOB) and $33 \mathrm{~dB}$ (5.2 ENOB) at near Nyquist input frequencies. Moreover, the slice channel shows less than $0.3 \mathrm{~dB}$ SNDR degradation compared to a single SAR in all the measured bandwidth. This means that the comparator offset calibration circuit works properly and no intra-slice offset mismatch is noted between the two sub-interleaved SAR ADC. Also, the TI-ADC achieves a peak SNDR of $33.9 \mathrm{~dB}$ (same as the slice) at low $F_{\text {in }}$ after the off-chip compensation of the gain and offset of the THA. A predictable SNDR degradation can be noted at high input frequencies due to sampling phase mismatch. However, after the manually delay cells adjustment on each sampling phase, an SNDR of $31.4 \mathrm{~dB}$ (4.92 ENOB) is achieved for the complete TI-ADC at $F_{s}=2 \mathrm{GS} / \mathrm{s}$.

For each SAR ADC, the power consumption is $3.3 \mathrm{~mW}$ at $1.2 \mathrm{~V}$ and the figure-of-merit defined as $F O M=$ Power $/\left(2^{E N O B_{N y q}} * F_{s}\right)$ is 0.63 (pJ/Conv-Step). The power consumption of the full TI-ADC is $192 \mathrm{~mW}$ (without considering VGA and Clock Phase Generator), while the overall FOM is $3.17(\mathrm{pJ} / \mathrm{Conv}-\mathrm{Step})$. The differential-non-linearity (DNL) and integral-non-linearity (INL) of the TI-ADC are 0.16 LSB and 0.49 LSB, respectively. Figure 8 shows the chip die micrograph.

\footnotetext{
${ }^{2}$ These measurements were done with the VGA bypassed.
}

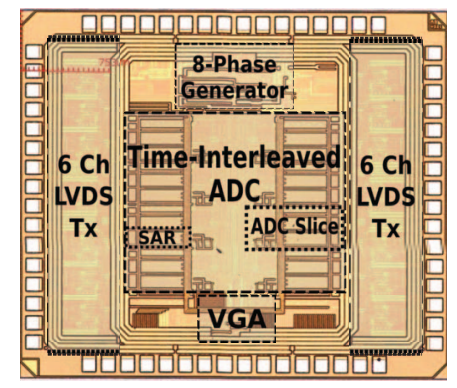

Fig. 8. Chip die photo. Die size: $3 \mathrm{~mm} \times 3.5 \mathrm{~mm}$.

\section{CONCLUSIONS}

A 6-bit 2-GS/s TI-SAR ADC has been designed, fabricated, and characterized. The implemented TI-ADC provides an excellent platform to evaluate mixed-signal calibration techniques, which are of great interest for application in high-speed optical systems.

\section{ACKNOWLEDGEMENT}

The authors would like to thank MOSIS for fabricating their design through the MEP research program.

\section{REFERENCES}

[1] W. J. Black and D. Hodges, "Time interleaved converter arrays," IEEE Journal of Solid-State Circuits, vol. 15, no. 6, pp. 1022-1029, 1980

[2] A. Nazemi et al., "A 10.3GS/s 6bit (5.1 ENOB at Nyquist) timeinterleaved/pipelined ADC using open-loop amplifiers and digital calibration in 90nm CMOS," in VLSI Circuits, 2008 IEEE Symposium on, 2008, pp. 18-19.

[3] A. Haftbaradaran and K. Martin, "A Background Sample-Time Error Calibration Technique Using Random Data for Wide-Band HighResolution Time-Interleaved ADCs," Circuits and Systems II: Express Briefs, IEEE Transactions on, vol. 55, no. 3, pp. 234-238, 2008.

[4] B. Razavi, "Design Considerations for Interleaved ADCs," IEEE Journal of Solid-State Circuits, vol. 48, no. 8, pp. 1806-1817, 2013.

[5] M. El-Chammas and B. Murmann, "A 12-GS/s 81-mW 5-bit TimeInterleaved Flash ADC With Background Timing Skew Calibration," Solid-State Circuits, IEEE Journal of, vol. 46, no. 4, pp. 838-847, 2011.

[6] C.-C. Huang et al., "A CMOS 6-Bit 16-GS/s Time-Interleaved ADC Using Digital Background Calibration Techniques," Solid-State Circuits, IEEE Journal of, vol. 46, no. 4, pp. 848-858, 2011.

[7] P. Schvan et al., "A 24GS/s 6b ADC in 90nm CMOS," in Solid-State Circuits Conference, 2008. ISSCC 2008. Digest of Technical Papers. IEEE International, 2008, pp. 544-634.

[8] B. Reyes, V. Gopinathan, P. Mandolesi, and M. Hueda, "Joint samplingtime error and channel skew calibration of time-interleaved ADC in multichannel fiber optic receivers," in 2012 IEEE International Symposium on Circuits and Systems (ISCAS), May 2012, pp. $2981-2984$.

[9] J. Cao et al., "A $500 \mathrm{~mW}$ ADC-Based CMOS AFE With Digital Calibration for $10 \mathrm{~Gb} / \mathrm{s}$ Serial Links Over KR-Backplane and Multimode Fiber," Solid-State Circuits, IEEE Journal of, vol. 45, no. 6, pp. 1172 $1185,2010$.

[10] U. Singh and M. Green, "Dynamics of high-frequency CMOS dividers," in IEEE International Symposium on Circuits and Systems, 2002. ISCAS 2002, vol. 5, 2002, pp. V-421-V-424 vol.5.

[11] J. Montanaro et al., "A 160-MHz, 32-b, 0.5-W CMOS RISC microprocessor," IEEE Journal of Solid-State Circuits, vol. 31, no. 11, pp. 1703-1714, 1996

[12] P. Figueiredo et al., "A 90nm CMOS 1.2v 6b 1GS/s two-step subranging ADC," in ISSCC 2006. Digest of Technical Papers. IEEE International, 2006, pp. 2320-2329.

[13] L. Kull et al., "A $3.1 \mathrm{~mW} 8 \mathrm{~b} 1.2 \mathrm{GS} / \mathrm{s}$ Single-Channel Asynchronous SAR ADC With Alternate Comparators for Enhanced Speed in $32 \mathrm{~nm}$ Digital SOI CMOS," IEEE Journal of Solid-State Circuits, vol. 48, no. 12 , pp. 3049-3058, 2013 